\title{
Optimization Control Based on Improved Traffic Signal Genetic Algorithm
}

\author{
Daming Li \\ School of Electronic Engineering, Wuhan Vocational College of Software and Engineering, Wuhan 430205, China
}

\begin{abstract}
To the optimization control problem of traffic signal timing of single intersection, an optimization method based on the Clustering Genetic Algorithm(CGA) was put forward. In a cycle, we take the total number of queue vehicles of corresponding release lane at the end of each phase as the performance index to construct the objective optimization function. The experiment results show that the total number of queue vehicles when CGA is applied to optimize is less than the ones when standard genetic algorithm(SGA) and traditional method are applied respectively; thus this method can effectively reduce the number of queue vehicles at the end of green light, and improve the traffic capacity of single intersection.
\end{abstract}

Keywords—single intersection; signal timing; genetic algorithm; clustering; the number of queue vehicles; objective function

\section{INTRODUCTION}

Traffic congestion is becoming a common problem for most urban areas. Thus, there is a need for effectively managing traffic signal control system. The control problem of traffic signal in urban road is one of basic problems of urban traffic management. Many traffic signal control system, such as TRANSYT, SCAT, and SCOOT, have been developed and applied[1].The single intersection is as the basic control object, so it is necessary to study it. There have been a lot of researches focused on signal timing of single intersection[2,3].

Due to the nonlinear, vagueness. and uncertainty of traffic flow, the optimization problem of signal timing of traffic intersection is usually considered a non convex nonlinear problem. So the traditional mathematical optimization method, such as algebraic method, graphic method and so on, cannot find the global optimal solution of problem above. Gnenetic algorithm(GA) is a kind of search technology based on natural selection and evolution, and is widely used to solve complex optimization problems, which also includes the optimization problem of signal timing of traffic signal[4,5].But they mostly adopted SGA as the optimization method, which has poor local search ability and is easy to bring out premature convergence. So in this paper, the SGA is improved by integrating clustering analysis firstly, and in a cycle. we take the total number of queue vehicles of corresponding release lane at the end of each phase as the optimization goal, the new method is applied into the optimization problem of signal timing of single intersection. Under the conditions of the random fluctuation of traffic flow, the new method can get the best optimal signal timing than the others.

\section{ThE IMPROVED GENETIC AlgORITHM BASED ON CLUSTERING ANALYSIS}

Genetic algorithm is a kind of search technology based on natural selection and evolution, and widely used to solve complex optimization problems. But the SGA is easy to fall into local optimal solution, bring premature convergence[6].In order to overcome the problem, wo integrate the clustering analysis into the GA. It can avoid inbreeding betweeen individuals by prohibiting individuals that belong to the same cluster from participating genetic operation. In the following sections, we first give a brief introduction of clustering analysis, then present the improved genetic algorithm based on clustering analysis.

\section{A. Clustering Analysis}

The clustering analysis is a kind of technology that divides a data set according to the difference of data attribute. And there are many types of clustering analysis methods, such as hierarchical clustering, k-means clustering, grid clustering, density clustering, etc. In this paper, we use the adaptive minimum spanning tree clustering method.

Assuming that the number of individuals in data sets is $n$, the steps of adaptive minimum spanning tree clustering algorithm are as follows:

- Step1.Calculate the Euclidean distance between any two of all individuals and make it as the edge's weight, and constitute a weighted-unidirectional graph.

- $\quad$ Step2.Find the graph's minimum spanning tree using the Prim algorithm.

- $\quad$ Step3.Calculate the average weight $\mathrm{M}$ of the minimum spanning tree's n-1 edges, and find max weight that is less than $\delta^{*} \mathrm{M}$ in the minimum spanning tree as threshold value ( $\delta$ is a user-specified decimal that is greater than 0 and less than 1 ).

- $\quad$ Step4.Remove the edges whose weight is greater than the threshold value, and the minimum spanning tree becomes a forest.

- $\quad$ Step5.Depth-traverse the forest, and save each population's individuals.

\section{B. The Clustering Genetic Algorithm}

Because the common selection method, the proportional selection, which is easy to produce a number of similar offspring in next generation. It can lead to premature loss of population's diversity and easy to cause premature convergence phenomenon. For this problem, the improved 
selection operator based on clusterviduals, and thus restrain the premature convergence.

By combing minimum spanning tree clustering algorithm above, we propose the improved genetic algorithm based on clustering analysis. The detailed steps are as follows:

- Step1: Initialize the algorithm: set the population size Popsize, crossover probability Pc, mutation probability Pm, the chromosome length etc.

- Step2: Generate randomly the initial population, calculating the fitness of each individual.

- Step3: Use minimum spanning tree clustering method to cluster current population, classify each individual and get the best individual in each cluster.

- Step4: Adopt the proportional selection to choose two parent individuals and determine the clusters of two parent individuals are different.

- Step5: Make two individuals selected in step4 to take part in single-point crossover and mutation, and get two new offspring individuals.

- Step6: If the number of new individuals reaches the population size Popsize, turn step7, otherwise turn step4.

- Step7: If evolution process reaches the number of evolution generation or satisfies stop conditions, then quit; Otherwise turn step4.

We adopt the improved genetic algorithm based on the minimum spanning tree clustering to solve the optimization problem of signal timing of single intersection, then, we get the signal timing, and use it to traffic control.

\section{The Optimization Model of Traffic Signal timing}

In optimization problem based on genetic algorithm, the optimization function is crucial. In order to get result of contrast simulation experiment, we refer the contents of literature [5], to establish the performance function of signal timing, form the optimization model.

\section{A. Single Intersection Traffic Flow Model}

In the single intersection, we adopt three lanes which respectively correspond to turning left, turning right and going straight. And adopt four phases: east-west going straight and turning right, east-west turning left, north-south going straight and turning right, and north-south turning left, shown in Figure1:
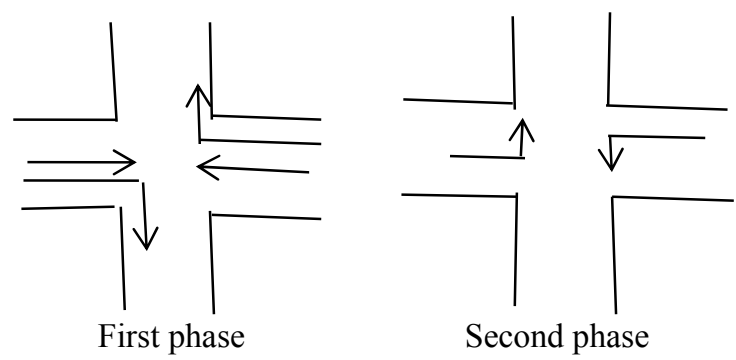

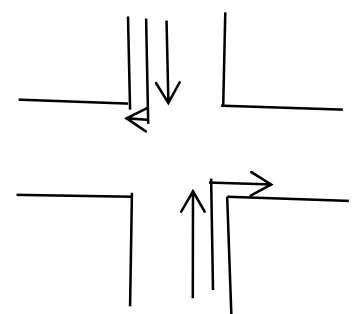

Third phase
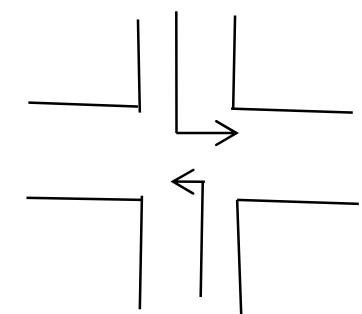

Fourth phase
FIGURE I. FOUR-PHASE DIAGRAM OF SINGLE INTERSECTION

Due to vehicle release status is different for different phase, different lane and different direction; a coefficient matrix $p_{e}$ is used to express the release status of intersection.

$$
p_{e}=\left\{p_{i j k}\right\}
$$

Where $\mathrm{i}$ indicates the phase, value range $1-4$, shown in Figure 4; indicates the direction, value range $1-4 ; \mathrm{k}$ indicates the lane, value range 1-3.

In this coefficient matrix, we make the value of zero represent correspond lane is forbid release and the value of one represent correspond lane is allow release. For instance, when p111=0, it means that at the first phase, the turning left lane of driving to east is forbid release.

\section{B. The Desing of CGA}

\section{1) Coding and Genetic Operation}

Because the signal control of single intersection need to get the green time of each phase, that is ti, a binary string is used to represent the signal timing of each phase. Since the sun of the signal timing is the cycle $\mathrm{T}$, when the cytle $\mathrm{T}$ is fixed, in order to get effective individual in the process of genetic operation, the binary string can only contain $t 1, t 2, t 3$, the $t 4$ can be obtainde by $\mathrm{T}$ subtract the other timing. So, the form of chromosome is $<\mathrm{t} 1 \mathrm{t} 2 \mathrm{t} 3>$. The shortest green timing of each phase should satisfy a certain threshold e, and the range of $e$ is $[\mathrm{e}, \mathrm{T}-3 \mathrm{e}][5]$.

The genetic operation about crossover and mutation adopt the common method single point crossover and anti-mutation, respectively. The selection operation is the clustering selection that was proposed in this paper[6].

\section{2) Objective Function}

In this paper, we use the improved genetic algorithms to optimize the signal timing of the single intersection. We establish the objective function as follows: In a cycle, at the end of each phase we take the total number of queue vehicles of corresponding release lane at the end of each phase as the optimization goal, and design the code according to the above paper, we get the calculate function of the fitness function as following[7]: 


$$
\operatorname{mis}=\min \sum_{i=1}^{3} \sum_{j=1}^{4} \sum_{k=1}^{3} p_{i j k} *\left(\begin{array}{l}
S_{i j k}+\lambda_{i j k} * t_{i}+\lambda_{i j k} *\left(T-\sum_{i=1}^{3} t_{i}\right. \\
-u_{i j k} * t_{i}-u_{i j k} *\left(T-\sum_{i=1}^{3} t_{i}\right)
\end{array}\right)
$$

Where $\min \mathrm{S}$ is the total number of queue vehicles. $S_{i j k}^{l}$ is the number of queue vehicles for $\mathrm{i}$ phase, $\mathrm{j}$ direction, and $\mathrm{k}$ lane at the end of cycle $1, t i(i=1,2,3,4)$ denotes the timing of phase $i$ of intersection, the four timing must satisfy with the sum is the cycle $\mathrm{T}$ and each timing has the range $[\mathrm{e}, \mathrm{T}-3 \mathrm{e}], \lambda_{i j k}$ and $u_{i j k}$ respectively express the vehicle arrival rate and the vehicle release rate for $\mathrm{i}$ phase, $\mathrm{j}$ direction, and $\mathrm{k}$ lane. $p_{i j k}$ is the release status.

\section{SIMUlation AND EXPERIMENT RESUlT}

The fitness evaluation function is formula (2), by solving it through use CGA to obtain the optimal solution ti. For the program, the operating parameters of genetic algorithm and traffic control are as follow: Group size is 100;termination of generation gen is 100 ; crossover probability is 0.8 ; mutation probability is 0.001 ;cycle length $\mathrm{T}$ is $120 \mathrm{~s}$;minimum green light time is $6 \mathrm{~s}$; the release vehicle rate $\mathrm{r}$ is $3500 \mathrm{~m} / \mathrm{h}$. Due to the vehicle arrival rate is not a stable value, it is up and down fluctuation, we randomly generate a value $\alpha r$ in range $[0.1 \alpha, \alpha r]$ ( $\alpha$ is a adjustment factor, to avoid the random value $\alpha r$ is too small, lose the significance).[8].

We use the VS2008 software to program the algorithm, and get the experiment result is shown in Table 1 .

TABLE I. THE DIFFERENT RESULTS WITH TRADITIONAL METHOD, SGA, AND CGA

\begin{tabular}{|c|c|c|c|c|c|c|c|c|c|c|c|c|c|c|c|}
\hline \multirow[t]{2}{*}{ Cycle } & \multicolumn{5}{|c|}{ Traditional(s) } & \multicolumn{5}{|c|}{ SGA(s) } & \multicolumn{5}{|c|}{ CGA(s) } \\
\hline & $\mathrm{t} 1$ & $\mathrm{t} 2$ & $\mathrm{t} 3$ & $\mathrm{t} 4$ & Smin & $\mathrm{t} 1$ & $\mathrm{t} 2$ & $\mathrm{t} 3$ & $\mathrm{t} 4$ & Smin & $\mathrm{t} 1$ & $\mathrm{t} 2$ & $\mathrm{t} 3$ & $\mathrm{t} 4$ & Smin \\
\hline 1 & 30 & 34 & 26 & 30 & 1 & 26 & 23 & 39 & 32 & 0 & 28 & 29 & 32 & 31 & 0 \\
\hline 2 & 35 & 27 & 28 & 30 & 25 & 36 & 18 & 36 & 30 & 6 & 35 & 23 & 32 & 30 & 6 \\
\hline 3 & 20 & 39 & 27 & 34 & 2 & 15 & 44 & 32 & 29 & 2 & 18 & 41 & 30 & 31 & 2 \\
\hline 4 & 16 & 50 & 24 & 30 & 9 & 15 & 29 & 35 & 41 & 0 & 16 & 39 & 30 & 35 & 0 \\
\hline 5 & 32 & 30 & 28 & 30 & 21 & 30 & 34 & 35 & 21 & 0 & 31 & 32 & 32 & 25 & 0 \\
\hline 6 & 32 & 30 & 28 & 30 & 13 & 34 & 44 & 33 & 9 & 2 & 33 & 37 & 30 & 20 & 1 \\
\hline 7 & 18 & 48 & 24 & 30 & 3 & 22 & 23 & 22 & 53 & 3 & 20 & 35 & 23 & 42 & 2 \\
\hline 8 & 28 & 40 & 22 & 30 & 12 & 26 & 32 & 23 & 39 & 4 & 27 & 36 & 23 & 34 & 1 \\
\hline 9 & 35 & 27 & 28 & 30 & 17 & 36 & 18 & 36 & 30 & 2 & 36 & 22 & 32 & 30 & 1 \\
\hline 10 & 30 & 34 & 26 & 30 & 1 & 27 & 24 & 38 & 31 & 1 & 29 & 29 & 32 & 30 & 1 \\
\hline
\end{tabular}

From this table, compared with the SGA and traditional method, the signal timing is better and more stable which get from use the the CGA. It is effectively reduce the total number of queue vehicles at intersection and improve the traffic capacity of intersection. $[9,10]$

\section{CONCLUSION}

The contrast experimental result shows that, the improved genetic algorithm can get more reasonable signal timing than both traditional method and the standard genetic algorithm, and thus it can significantly reduce the total number of queue vehicles at intersection and improve the traffic capacity of intersection. In this paper, we only consider the motor vehicle, did not consider pedestrians and other factors. In actual application, the factors that can influence traffic should be considered. While this article aims at signal timing of single intersection with four-phase, it can be extended to the application of multiple intersections with multiple phases. At the same time, this article uses the number of queue vehicles as performance index, which can alse use other indicators, such as: the vehicle delay, stops, etc.[11,12].

\section{ACKNOWLEDGEMENTS}

In this paper, the research was sponsored by the Education Department Foundation of Wuhan of China under Grant No. 2012038.

\section{REFERENCES}

[1] Z. Miao, K. Fu, and F. Yang, "A hybrid genetic algorithm for the multiple crossdocks problem," Mathematical Problems in Engineering, vol. 2012, Article ID 316908, 18 pages, 2012. View at Publisher ·View at Google Scholar.

[2] Li Xiangsheng, Traffic signal optimization control and simulation of green split in a intersection using genetic algorithm method. Journal of Central South University of Forestry \& Technology,307(7),pp.155159,2010 .

[3] Li Yujian. Minimal spanning tree cutting algorithm. Journal of Beijing University of Technology,2006.

[4] Wang Wei, Guo Xiucheng. Traffic engineering .Nanjing: Southeast University Press,2000.

[5] Wan Wei, Chen Feng, Optimal control for isolated intersection signal based on genetic algorithm. Computer Engineering,33(16),pp.217$219,2007$.

[6] Chen Guoliang, Genetic algorithm and application. Beijing: People's Posts and Telecommunications Press, 1996.

[7] Xu Zhongmin, He Yansong. Foreign research trends of intelligent transportation system. The Automotive Industry Research, No 3,2001,pp.34-38.

[8] Zhang Mengmeng, Jia lei, Zou Nan, Robust optimal traffic signal timing of urban single-point intersection. Computer Engineering,37(6),pp.2729,2011 .

[9] Mohannad Sadegh Norouzzadeh, Plowing PSO: A Novel Approach to Effectively Initializing Particle Swarm Optimization. Computer Science and Information Technology (ICCSIT), 2010 3rd IEEE International Conference: $705-708$. 
[10] He Zhaocheng, Yang Wewei, Sun Jian,Dong Lin,Real-time traffic signal timing program of single junctions. Journal of Shanghai University of Engineering Science,23(4),pp,291-296,2009.

[11] M.pant, Thangarag R., and A Avraham," Imporved Particle swarm Optimization with Low-discrepancy Sequences," in IEEE Cong. on Evolutionary Computation(CEC), Hong Kong, 2008.

[12] Hbabaee, An improve PSO based Hybrid Algorithms. Management and Service Science(MASS), International Conference, 2011. 